\title{
Tackling the COVID-19 Pandemic: A Review on Way Forward
}

\author{
Vishali Dutta \\ Jawaharlal Nehru Medical College, Datta Meghe Institute of \\ Medical Sciences, Sawangi, Wardha, Maharashtra, India. \\ Corresponding author email: vishali.dutta@gmail.com
}

\section{ABSTRACT}

Novel coronavirus which has been invented since a year has wreaking havoc all over the world by infecting people with COVID-19 or coronavirus disease 2019. The COVID-19 is extremely virulent and can spread extremely fast. Bodily secretions, sneeze, cough and breath can give easy way to the virus to transmit from host to the primary contact. The incubation period lasts from two days to fourteen days. Symptoms includes. Fever, cough, fatigue, shortness of breath, body aches, headaches, sore throat, runny nose, loss of smell and taste, diarrhea, vomiting and many more. The global nature of the disease has provided vast scope for study especially symptoms as they vary greatly and with different combination. Entry points for the virus to enter into the body are eyes, nose, mouth etc. Respiratory systems are primarily the first affected. The containment of COVID-19 is primarily based on test, trace, treat model whose first element is test. Various tests are approved by concerned governing and health authorities to identify the patients infected of COVID-19. The test which is considered as golden standard for the diagnosis of the COVID-19 is Reverse Transcript Polymerase Chain Reaction (RT-PCR) test which works on the principle of ribonucleic acid (RNA) molecule amplification of coronavirus strain. Same test was also used in previous outbreak of severe acute respiratory syndrome (SARS) in 2002. A nasopharyngeal sample which is secretion present in the respiratory system or saliva and stool sample may collected to test it for the infection.More study is needed on different aspects of containing the coronavirus spread and limit transmission to halt the pandemic.

KEY WORDS: CORONA VIRUS, RESPIRATORY, COVID-19, STRAINS, DISTANCING, PREVENTION, TRANSMISSION.

\section{INTRODUCTION}

Novel coronavirus which has been invented since a year has wreaking havoc all over the world by infecting people with COVID-19 or coronavirus disease 2019. The crown shaped virus started its disastrous journey from the Wuhan city of the Hubei province of China (Dushyant Bawiskar,et. al. 2020). Since then now it has been a year and there is no sign of slowing down of the virus. In fact in some parts of the Europe more virulent strain which is believed to be mutated has been reported creating panic again all across the world. Till December

Biosc Biotech Res Comm P-ISSN: 0974-6455 E-ISSN: 2321-4007

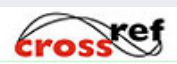

Identifiers and Pagination

Year: 2021 Vol: 14 No (6) Special Issue

Pages: 220-224

This is an open access article under Creative

Commons License Attribn 4.0 Intl (CC-BY).

DOI: $h t t p: / / d x . d o i . o r g / 10.21786 / b b r c / 14.6 .46$
23, 2020 78,018,199 infected cases has been reported of which 1,717,124 people had died due to COVID-19 complications. United States of America, India, Brazil, Russian federation, France and United Kingdom are top six countries that accounts for more than half of the world infection and death rates. United States of America and India had crossed the unfortunate mark of 10 million infected cases.

Whereas in UK the discovery of new and mutated strain of coronavirus reported to be more virulent than the present one has created a scare wave all across the globe. Comorbidity is one of the biggest culprit in increasing case fatality numbers. Diabetes, hypertension, renal failure, coronary artery disease accounts for majority of the case fatalities infected with COVID-19. Since its inception the virus has been able keep itself unpredictable that whenever the curve sees downward trend suddenly it gets shot up and complacency has no place in the containment strategy. The symptoms and pathophysiology

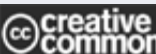

Article Information

Received: $29^{\text {th }}$ March 2021 ccepted after revision: $29^{\text {th }}$ May 2021 
has so farfluctuating among certain factors and needs to be monitored carefully. Various tests are being deployed to diagnose the people whenever necessary.

Some of them requires high sophistication while some can be done easily with less training and time. Error margin must be the decider as the more you are accurate less is the chance of missing any positive case as negative or vice versa. Early detection can be helpful in dampening the rate of infection. It is found out that including certain foods in diet containing specific vitamins and probiotics may strengthen the innate immune system which will help in fighting the invasion of COVID-19. Treatment of the COVID-19 may vary according to the individual's medical condition. A new phenomenon of post COVID-19 implications is gaining roots and may prove strenuous on health service sector. This is the reason why preventive measure are very important. In this article a comprehensive overview of all these factors will be taking place.

Pathophysiology of Covid-19: Like other virus strains, coronavirus also works on the principle of enter and multiply and then take the control of the host cell and disturb the functions of the cell. As it is named on its crown like structure, it has spike or spiky surface proteins which then gets attached to the healthy receptors of the cell, entered through nose, mouth or eye. The gateway through which it enters the healthy cell is angiotensin enzyme 2 receptors or ACE2 receptors (Phadke M, Saunik $S$ 2020). Then it takes control of the cell and kill some of the healthy cells. The COVID-19 is extremely virulent and can spread extremely fast. Bodily secretions, sneeze, cough and breath can give easy way to the virus to transmit from host to the primary contact. The incubation period lasts from two days to fourteen days. Symptoms includes. Fever, cough, fatigue, shortness of breath, body aches, headaches, sore throat, runny nose, loss of smell and taste, diarrhea, vomiting and many more.

The global nature of the disease has provided vast scope for study especially symptoms as they vary greatly and with different combination. Entry points for the virus to enter into the body are eyes, nose, mouth etc. Respiratory systems are primarily the first affected and also lower respiratory tract contains more number of ACE2 receptors which further facilitates the entry of the coronavirus in to the cell. The viral load shifts downward from upper thoracic region to lungs and then transmitted to different parts of the body. Inflammation is primary response to such invasion of external pathogen and lungs can show such symptoms affecting regular and proper functioning of the respiratory system. Which may lead to pneumonia which is related to alveoli which is essential to throw out the harmful carbon dioxide gas and diffuse the oxygen onto blood vessels (Becker RC.2020). A computed tomography (CT) scan can be done to see patches that indicates the improper functioning of the alveoli.

Most of the cases are of mild nature and show moderate symptoms. If infection persists more than five days without getting medical intervention then chances of developing short ness of breath or dyspnea followed by acute respiratory distress syndrome (ARDS). ARDS can cause sweating, rapid heart rate, increased breathing rate resulting in damage of tissues and blood vessels in alveolar region. Then chance of ventilator support increases manifold especially in ARDS case. As debris and fluid stats to gather in lungs, it is then become more difficult to provide oxygen to various parts of the body and vital for proper functioning of the body.

Detection of Covid-19: The containment of COVID-19 is primarily based on test, trace, treat model whose first element is test. Various tests are approved by concerned governing and health authorities to identify the patients infected of COVID-19. The test which is considered as golden standard for the diagnosis of the COVID-19 is Reverse Transcript Polymerase Chain Reaction (RT-PCR) test which works on the principle of ribonucleic acid (RNA) molecule amplification of coronavirus strain. Same test was also used in previous outbreak of severe acute respiratory syndrome (SARS) in 2002 (Ngai JC, et. al. 2010). A nasopharyngeal sample which is secretion present in the respiratory system or saliva and stool sample may collected to test it for the infection. Test checks for two genes in the sample, if both of them found then the samples is said to be tested positive for COVID-19, if only one sample is found then it is said to be inconclusive and if none is found then it is given as negative in reports.

To see the expanse and spread of the virus in lungs, the CT scan of lungs may be advised in order to have a proper idea about the infection that is being spread in the patient's body. RT-PCR can take a day or two to reach some conclusion (Hendren NS, et. al. 2020). Also it is a very sophisticated test therefore it requires trained professionals and proper machinery. In asymptomatic patients or patients with mild to none symptoms and are in initial phase of the infection, the viral load may be less and the sample efficacy is affected in negative way. Whereas in some cases the viral load is shifted to lower end of the respiratory tract and may not be sampled properly. In some cases human error may also contribute to false result obtained from the test. In some places, there are point of care units that test the sample and give quicker results, almost in an hour. It is widely used in high vulnerable places such as hospital, and used for health care professionals so that they get tested on urgent basis as they have to treat the COVID-19 patients.

Then there are antibodies test which checked in the suspected individuals blood samples. If particular antibodies are found then they are said to be infected with coronavirus. It is used to check the disease prevalence in a particulararea and helps in curating the strategy targeted to that specific area utilizing the resources judiciously. Due to huge population coverage of the disease and same number are vulnerable, various governing agencies devised questionnaire based selfassessment test. It is basically set of question that one has to ask to itself (Meo SA,et. al. 2020). If it is seen that the infection is suggested then one has to report to nearest 
COVID-19 center and get them tested otherwise there is no need to test as there are already lot of application and samples needs to be assessed so avoiding unnecessary testing will only help agencies and save time as well as money.

Dedicated COVID-19 facilities along with testing centers have been established to deal with the pandemic with war mode activated. The devastating effect suggests more facilities needs to be built so that pandemic can be contained in effective and time bound manner. Post ill ness implications are also in consideration as was the case in SARS and MERS outbreaks. Health infrastructure strengthening is the need of the hour and will persist long time even after pandemic is over (Sarma P, et.al. 2020).

Prophylactics: The crumbling of health care infrastructure was seen not only in least developed and developing nations but also in developed countries aswell. It was attributed to virus's high virulent nature and its deadly health consequences. Current health infrastructure is overwhelmed by the pandemic and needs alternative strategy to deal with. Preventive aspect of the disease is more suitable than the curative one and can serve many purpose, benefitting both health care authorities and individuals as well. A year into the pandemic which was originated in Wuhan, China, some trends have been confirmed such as comorbidity can be decider of the severity of symptoms in COVID-19 infected patients, long term implications of COVID-19 may include persistence of the symptoms such as fatigue and myocarditis and so on.

Therefore building immunity and following rules are the only options left for the people to stay away from the virus and which are not expensive and easy to follow. Chronic underlying medical conditions or comorbidities are saidto be the biggest influencer in COVID-19 infection outcomes. Major chunk of the mortalities up to 70 percent are due to comorbidity conditions that were proving hindrance in the treatment of the COVID-19 (Hussain A, et. al. 2020). Maintaining a healthy lifestyle can help us to stay fit and healthy. Eating proper and balanced diet may also prove benefitting the overall wellbeing of the person. Certain foods containing Vitamin C, Vitamin Dand probiotics can be inculcated in the diet to improve the functioning of the body (Feyaerts AF, Luyten W. 2020). They may be taken as foods and vegetables or supplemented externally in the form of tablets and pills. Vitamin c is proven to be reduce inflammatory condition and reduce oxidative stress induced in body.

Vitamin $\mathrm{d}$ is not only associated with absorption of the calcium in the body but has direct connection with the immune system of the body (Pizzini A,et. al. 2020). So having it in adequate form especially when there is prevalence of deficiency of vitamin $d$ all across the globe, will only help in metabolism. There is a famous gut lung connection. If the number of good bacteria lessens then it directly affects the lung and immunity of the body. Probiotics are meant to maintain the sufficient amount of good bacteria that are essential for digestion and absorption of food components (Sundararaman A, et. al. 2020). A study found that people having yoghurt in their diet, which is full of good bacteria and act as replenishment of them, fall less ill suggesting better immunity than their non-eating counterparts.

The long term implications of the disease has been started to surface as it was bound to happen as it was also happened in previous outbreaks of SARS and MERS. It includes fatigue, loss of taste and smell, dyspnea, weakening of heart muscles and so on. Already the current health infrastructure is not able to bear the load of the incoming patient, it will be difficult to accommodate the patients complaining from post illness disorder syndrome. Therefore awareness campaign about being safe and taking utmost precaution to safeguardyourself from the infection should be started. Necessary foods list that are in generalno harmful to anybody and can act as prophylactics can be publically released in order to acquaint people to them. Fortification of foods under government schemes may also work as some farfetched regions can be provided with them.

Treatment of Covid-19: There are two types of care post infection which are being followed primarily across the globe. Almost majority of the young population need not even show symptoms if they caught the infection of the COVID-19. If symptoms are being shown they will be in most cases of mild in nature and can be easily treated with isolation and rest formula. Proper precaution is necessary to safeguard others who are there to help the infected person help in daily chores at home. Wearing of masks, personal and protective equipment'sshould be worn to avoid getting infected. One with mild symptoms and infection of COVID-19 should rest which can hasten up the recovery process. Staying home isolated would be another step in treatment of COVID-19 at home. Keeping one's body hydrate by replenishing it with fluids is also essential for the cure. Be in contact with the doctor continuously and if slight worsening of the symptoms is seen then medical intervention must be sought (Liu X, et. al. 2020).

The treatment at the hospital is more sophisticated and under continuous monitoring which is required in patients having severe symptoms. Oxygen levels, in blood and lungs, heart rate, CT scan can be done to assess the situation properly. Regular testing if the stay at the hospital is extended then is done to check on the status of the disease. Provision of ventilator, oxygen support system has been done to ensure proper treatment of critically ill patientsespecially elders who are most vulnerable in current pandemic scenario. The course and medicinal treatment may vary according to patient as it depends on severity of the disease. General administration of vitamins and antibiotics are being done as there is no predefined set of instruction on administration of drugs. No self-administration of drugs which so ever should be done without the consultations of the doctor or competent authority to avoid any medical complications (Mason RJ. 2020). 
Post Covid-19 Care: The case for reinfection of COVID19 infection got strengthen when certain number of individuals claimed that they got the infection even after they got treated for it. The persistence of the antibodies in the blood is the topic to study as there is no information up to how many days the antibodies persist in the blood. Also incomplete treatment, re exposure to personnel's like doctors and nurses can be the possible reasons behind the supposedly reinfection. The long term implication of the COVID-19 has been underplayed till now, but as soon as the number treated patients started to complain about their symptom post discharge then we have to study more in to the matter. Mostly elderly who survived the infection are finding it difficult to cope up with post illness period as their overall capacity is reduced. Not just elderly but normal people after infection are showing and complaining about the reduction in physical capacity, fatigue, intermittent loss of taste and smell etc.

After clinically examining these patients, it is found out that not only these but effects are profound on vital internal organs such as kidney, heart, neurological system, pulmonary system and so on. Weakening of cardiovascular muscles, myocarditis, reduced capacity of diffusion of carbon monoxide outside the body (DLCO), blood vessel damage, brain fog which is difficulty in decision making ability at times. Therefore the need of the dedicated post-COVID-19 care centers may arise in future as the cases of post illness disorder are on rise. Various departments of the medical organization needs to collaborate in order to tackle the post illness disorder because the post illness disorder are various and concerned to different branches of medicine (Rimmer A.2020 and Toshida, T., and Chaple J.2020).

Preventive Measures: Various measures can be employed to prevent the infection from happening. World Health Organization (WHO) along with various governmental agencies has released the guidelines about proper preventive measures which can be undertaken to break the chain of infection. A number of related studies were reviewed (Ather B et al 2020). Initially, at the start of the pandemic the blanket measure of lockdown and physical distancing was imposed to contain the spread of the infection. It was not very effective as cases hot up as soon as the lockdown started to ease up (Gaidhane S, 2020 Jachak, S.,2020 Joseph, M.B. 2020). But it bought some time to ramp up the health care facilities to deal with the pandemic.

Then reliance on non-pharmacological interventions like wearing of mask, gloves,face shields, appropriate PPE kit, maintaining minimum safe distance were popularized owing to their benefits, which was also seen in previous outbreaks of SARS and MERS (Joshi, K., 2020, Khatib, M.N., 2020 and Lakhkar, B.B., 2020). Also in Ebola outbreak in African continent, it was only possible through PPE kit that allied health care professionals were able to trace the patient without getting infected as there were already a lack of health care professionals. In COVID-19 also, health care professionals are already at high risk as they are the one treating the patients infected from COVID-19 (Somashekhar, S. P., et. al. 2020 and Dhankasar, 2021).

Their safety should be of utmost priority. Already the number of doctors or nurses per unit population is not sufficient all over the world. Therefore we cannot afford to lose them at any cost. They must be provided with sophisticated and adequate amount of PPE kit with appropriate fitting which can be customize so that they remain infection free and can treat patients fearlessly. Already according to international council for nurses, there has been more casualties of nurses during COVID19 duty than First World War which is quite alarming. Therefore necessary precaution needs to take in order to protect the health care professionals and in general all the people to remain infection free.

\section{CONCLUSION}

A lot has been known about COVID-19 and same amount is remain unearthed. Uncertain nature of the coronavirus is what it masks so deadly. The grave nature can be even from the casualty numbers with case fatalities crossing a million mark and not yet controlled. Vaccine is in final stage but its distribution and study of efficacy remains unstudied and needs time. Therefore it is important to plan for at least for considerable amount of time as we have to live with this virus. Possible treatment needs dynamic changes in COVID-19 tines. Long term implications has seriously asked for ramping up the health infrastructure to support the post COVID-19 rehabilitation cases. Also preventive measures can be inculcated in order to prevent the disease from happening at the first place. Especially medical professionals who are at high risk of getting contracted with virus. Finally we are what we eat, therefore by improving our lifestyle we can beat not only this pandemic but also be future ready as there can be one in future too.

\section{Conflict of Interest: NIL}

\section{Funding: DMIMS(WARDHA)}

\section{REFERENCES}

Acharya, S., Shukla, S. and Acharya, N., 2020. Gospels of a pandemic-A metaphysical commentary on the current COVID-19 crisis.

Ather, B., Mirza, T.M. and Edemekong, P.F., 2020. Airborne precautions. StatPearls [Internet].

Bawiskar, D., Phansopkar, P. and Gotmare, A.V., 2020. Covid-19 facets: Pandemics, curse and humanity. International Journal of Research in Pharmaceutical Sciences, 11(Special Issue 1).

Becker, R.C., 2020. Covid-19 treatment update: follow the scientific evidence.

Dhankasar, P., Dhole, P., Kolhe, S., Dambare, M., Rewatkar, P. and Balpande, V., 2020. The Unseen Positive Effect of Covid-19 Pandemic. Journal of Research in Medical and Dental Science, pp.108-112. Feyaerts, A.F. and Luyten, W., 2020. Vitamin C as 
prophylaxis and adjunctive medical treatment for COVID-19?. Nutrition, 79, p.110948.

Fritz, Z., Huxtable, R., Ives, J., Paton, A., Slowther, A.M. and Wilkinson, D., 2020. Ethical road map through the covid-19 pandemic.

Gaidhane, S., Khatib, N., Zahiruddin, Q.S., Gaidhane, A., Telrandhe, S. and Godhiwal, P., 2020. Depression, anxiety and stress among the general population in the time of COVID-19 lockdown: A cross-sectional study protocol. International Journal of Research in Pharmaceutical Sciences, 11(Special Issue 1).

Guan, W.J., Liang, W.H., Zhao, Y., Liang, H.R., Chen, Z.S., Li, Y.M., Liu, X.Q., Chen, R.C., Tang, C.L., Wang, T. and Ou, C.Q., 2020. Comorbidity and its impact on 1590 patients with COVID-19 in China: a nationwide analysis. European Respiratory Journal, 55(5).

Gunawan, J., Aungsuroch, Y., Marzilli, C., Fisher, M.L. and Sukarna, A., 2021. A phenomenological study of the lived experience of nurses in the battle of COVID-19. Nursing outlook.

Hendren, N.S., Drazner, M.H., Bozkurt, B. and Cooper Jr, L.T., 2020. Description and proposed management of the acute COVID-19 cardiovascular syndrome. Circulation, 141(23), pp.1903-1914.

Hussain, A., Bhowmik, B. and do Vale Moreira, N.C., 2020. COVID-19 and diabetes: Knowledge in progress. Diabetes research and clinical practice, p.108142. Islam, M.S., Sarkar, T., Khan, S.H., Kamal, A.H.M., Hasan, S.M., Kabir, A., Yeasmin, D., Islam, M.A., Chowdhury, K.I.A., Anwar, K.S. and Chughtai, A.A., 2020. COVID19-related infodemic and its impact on public health: A global social media analysis. The American Journal of Tropical Medicine and Hygiene, 103(4), p.1621.

Jachak, S., Phansopkar, P. and Naqvi, M.W., 2020. Impact of covid-19 in India, a disastrous pandemic outbreak. International Journal of Research in Pharmaceutical Sciences, 11(Special Issue 1).

Joseph, M.B., Pohekar, S., Raut, A. and Patil, M., 2020. The palliative care and covid-19 pandemic. International Journal of Research in Pharmaceutical Sciences, 11(Special Issue 1).

Joshi, K., Acharya, N., Acharya, S. and Joshi, S., 2020. A grave situation with COVID in the gravid: A narrative review. International Journal of Research in Pharmaceutical Sciences, pp.496-499.

Khatib, M.N., Gaidhane, S., Khatib, M., Ahmed, M., Gaidhane, A. and Syed, Z.Q., 2020. SARS-CoV and SARS-CoV-2: Similar viruses with different trajectories. Wutan Huatan Jisuan Jishu, 16(5).

Lakhkar, B.B., Guru, B., Damke, S. and Damke, S., Most Susceptible Duo in COVID-19 Crisis: A Literature Review. Perinatology, 21, pp.112-23.

Latchoumi, T.P., Ezhilarasi, T.P. and Balamurugan, K., 2019. Bio-inspired weighed quantum particle swarm optimization and smooth support vector machine ensembles for identification of abnormalities in medical data. SN Applied Sciences, 1(10), pp.1-10.
Liu, X., Liu, C., Liu, G., Luo, W. and Xia, N., 2020. COVID-19: Progress in diagnostics, therapy and vaccination. Theranostics, 10(17), p.7821.

Mason, R.J., 2020. Pathogenesis of COVID-19 from a cell biology perspective.

Meo, S.A., Klonoff, D.C. and Akram, J., 2020. Efficacy of chloroquine and hydroxychloroquine in the treatment of COVID-19. Eur Rev Med Pharmacol Sci, 24(8), pp.45394547.

Ngai, J.C., Ko, F.W., Ng, S.S., TO, K.W., Tong, M. and Hui, D.S., 2010. The long-term impact of severe acute respiratory syndrome on pulmonary function, exercise capacity and health status. Respirology, 15(3), pp.543550.

Phadke, M. and Saunik, S., 2020. COVID-19 treatment by repurposing drugs until the vaccine is in sight. Drug development research, 81(5), pp.541-543.

Pizzini, A., Aichner, M., Sahanic, S., Böhm, A., Egger, A., Hoermann, G., Kurz, K., Widmann, G., Bellmann-Weiler, R., Weiss, G. and Tancevski, I., 2020. Impact of vitamin $\mathrm{d}$ deficiency on COVID-19-A prospective analysis from the CovILD registry. Nutrients, 12(9), p.2775.

Rimmer, A., 2020. Covid-19: Impact of long term symptoms will be profound, warns BMA.

Sarma, P., Prajapat, M., Avti, P., Kaur, H., Kumar, S. and Medhi, B., 2020. Therapeutic options for the treatment of 2019-novel coronavirus: An evidence-based approach. Indian journal of pharmacology, 52(1), p.1.

Singh, K.T., Mishra, G., Shukla, A.K., Behera, S., Tiwari, A.K., Panigrahi, S. and Chhabra, K.G., 2020. Preparedness among dental professionals towards COVID-19 in India. The Pan African Medical Journal, 36.

Singh, N., Anjankar, A.P. and Garima, S., 2020. The Urgent Need to Understand Covid-19 Associated Coagulopathies and the Significance of Thrombotic Prophylaxis in Critically Ill Patients. Journal of Evolution of Medical and Dental Sciences, 9(33), pp.2381-2386.

Shah, P. and Naqvi, W., 2020. Fighting and chasing the rogue virus-covid 19. International Journal of Research in Pharmaceutical Sciences, 11(Special Issue 1).

Somashekhar, S.P., Shivaram, H.V., Abhaham, S.J., Dalvi, A., Kumar, A., Gode, D., Misra, S., Jain, S.K., Prasad, C.R.K. and Pillarisetti, R.R., 2020. ASI's consensus guidelines: $\mathrm{ABCs}$ of what to do and what not during the COVID-19 pandemic.

Sundararaman, A., Ray, M., Ravindra, P.V. and Halami, P.M., 2020. Role of probiotics to combat viral infections with emphasis on COVID-19. Applied microbiology and biotechnology, pp.1-16.

Thorlund, K., Dron, L., Park, J., Hsu, G., Forrest, J.I. and Mills, E.J., 2020. A real-time dashboard of clinical trials for COVID-19. The Lancet Digital Health, 2(6), pp.e286e287.

Wise, J., 2020. Covid-19: New coronavirus variant is identified in UK 